\title{
Vehicular Traffic Management Using Cloud Network App
}

\author{
Jayshree Dayanand Mallapur ${ }^{1}$, Renuka Takappa Ambiger ${ }^{2}$ \\ ${ }^{1}$ Department of Electronics and Communication Engineering, Basaveshwar Engineering College, Bagalkot, India \\ ${ }^{2}$ Department of Computer Science and Engineering, Head of Department, B. V. V. S. Polytechnic, Bagalkot, India
}

\section{Email address:}

renukaran@yahoo.com (R. T. Ambiger)

\section{To cite this article:}

Jayshree Dayanand Mallapur, Renuka Takappa Ambiger. Vehicular Traffic Management Using Cloud Network App. International Journal of Science, Technology and Society. Vol. 5, No. 6, 2017, pp. 203-209. doi: 10.11648/j.ijsts.20170506.15

Received: September 30, 2017; Accepted: October 20, 2017; Published: November 29, 2017

\begin{abstract}
In cities around the world commuters complain that traffic congestion makes the travel inconvenient and arduous. Vehicular networking and communication system is an area of significant importance. In our increasingly connected and mobile world, he state of road density is an important factor in evaluating travel times and travelling routes. Effective vehicular connectivity techniques can significantly enhance efficiency of travel, reduce traffic incidents and improve safety, mitigate the impact of congestion and overall provide more comfortable experience. Our solution delivers cloud approach to the traffic management by pulling information about the traffic density in the road junction. This translates to improved service by reducing unnecessary stops and elevates the comfort in travel.
\end{abstract}

Keywords: Communication, Vehicular Networks, Cloud Approach, Internet of Things

\section{Introduction}

In any city in the world, traffic monitoring is an important part of the smart-city infrastructure. Normal traffic to highway traffic requires adequate information about the support and logistics available on the highway and in turn the system can be made self-reliable and intelligent. Any type of congestion on roads will ultimately lead to loss of fuel and economic loss. Any foresight on traffic will always help to improve the whole system. With number of WSN and Sensor enabled communications, an IoT of traffic will be generated. This will be known as Traffic IoT (TIoT). The information collected from TIoT can be presented to travelers as in [4].

The traffic information will be dependent upon the queuing model on roads and infrastructure of roads itself. This identification of critical road points and present state of traffic information on all roads can be provided to the user. However, this traffic monitoring application needs to be secure to prevent any terrorist attacks frequent in urban cities. Few such prototype implementations can be found in [8][9] and the Smart Santander EU project [11].

\subsection{Internet of Things (IoT)}

The term 'Internet of Things' or 'Internet of Objects' has come to represent electrical or electronic devices, of varying sizes and capabilities that are connected to the Internet. The scope of the connections is ever broadening to beyond just Machine-to-Machine communication (M2M). IoT devices employ a broad array of networking protocols, applications and network domains [1]. The rising preponderance of IoT technology is facilitated by physical objects being linked to the Internet by various types of short-range wireless technologies such as ZigBee, RFID, sensor networks and through location based technologies. IoT will make the impact of the Internet even more pervasive, personal and intimate in daily life. The emergence of IoT as a distinctive entity was achieved, according to the CISCO Internet Business Solutions Group (IBSG), when more inanimate objects were connected to the Internet than human users. According to this definition, this occurred in mid-2005. This is an accelerating ongoing process especially with the rollout of CISCO's 'Planetary Skin', the Smart Grid and intelligent vehicles.

IoT devices are not currently strongly standardized in how they are connected to the Internet, apart from their networking protocols. IoT may be employed with added management and security features to link as in vehicle electronics, home environmental management systems, telephone networks and control of domestic utility services. The expanding scope of IoT can be used to interconnect 
various disparate networks.

\subsection{Internet of Things Architecture}

As identified by Atzori et. al. [3], Internet of Things can be realized in three paradigms - Middleware, Sensors and the Knowledge Base to interact and fulfill the visions of internet oriented, things oriented and semantic oriented. Individual visions will not fulfill the whole connectivity vision. The intersection of these visions will be main focus for making and connecting the objects in to the network. For any network, things will be the active participants in business, information and social processes. Figure 1 illustrates the IOT Architecture.

We have thought of giving more focus on the user requirements of the sensors and smartness to enable IoT for sensible smart environments with any interconnections of sensors and actuators that have ability to process raw sensor information, and meaningful inferences to develop a unified picture of the situation for smart applications. This is possible through large scale sensor networks, data processing and inferences using smart sensing and cloud computing. The inferences will make the sensors sensible enough to rectify the whole process which in turn will move towards making the whole process intelligent.

\subsection{Wireless Sensor Networks}

The components that make up the WSN monitoring network include:

Wireless Sensor Networks hardware - Typically a WSN node contains interfaces to sensors, computing and processing units, transceiver units and power supply. More sophisticated sensor nodes can communicate over multiple frequencies as stated in [2].

Wireless Sensor Networks Communication Stack (WSNCS) - The nodes will be deployed in an adhoc manner.

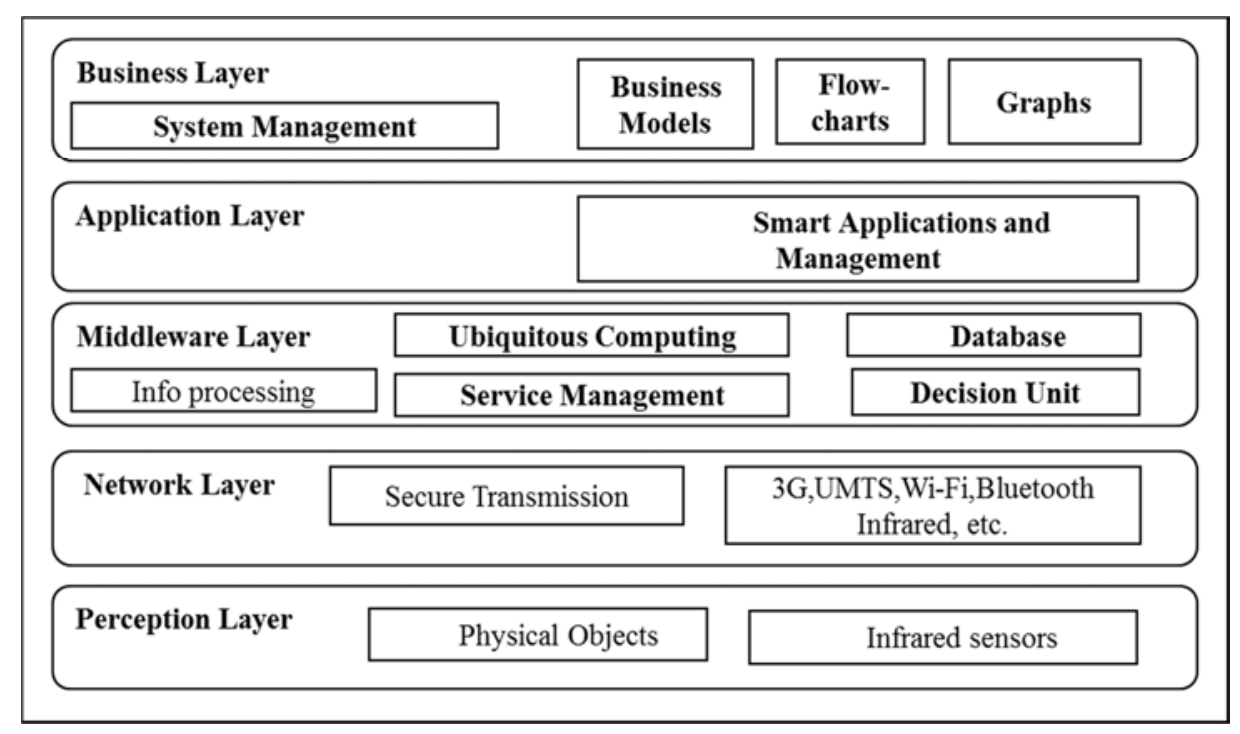

Figure 1. The IoT Architecture.

Communication topology will be an important factor for communication through the system of WSN nodes. There is this communication stack at one central node which will be able to interact with the connected world through the Internet and which will act as a gateway to the WSN subnet and the Internet [9].

Middleware-This is associated with the internet infrastructure and the concept of service oriented architecture (SOA) for access to heterogeneous sensor resources as described in [5].

\subsection{Cloud Computing}

Cloud computing is an emerging paradigm of distributed computing. In 1969 [14] L. Kleinrock anticipated, "As of now, computer networks are still in their infancy. But as they grow up and become more sophisticated, we will probably see the spread of 'computer utilities' which, like present electric and telephone utilities, will service individual homes and offices across the country." His vision was the true indication of today's utility based computing paradigm. One of the giant steps towards this world was taken in mid1990'swhen grid computing was first coined to allow consumers to obtain computing power on demand. The origin of cloud computing can be seen as an evolution of grid computing technologies. The term Cloud computing was given prominence first by Google's CEO Eric Schmidt in late 2006 (may be he coined the term) [15]. So the birth of cloud computing is a very recent phenomena although its root belongs to some old ideas with new business, technical and social perspectives. Cloud uses the existing grid architecture to provide services and add-ons few technologies like virtualization and various business models.

In brief, cloud computing is a cluster of commodity of computer networks in different geographical locations or the same geographical location, operating together to serve a number of customers with different need and workload on demand basis with the help of the virtualization. Cloud services are provided to the cloud customers or clients as 
utility services like electricity, telephone as pay-as-you-use business model. These utility services are generally described as the Xaa $\mathrm{S}$ ( $\mathrm{X}$ as a Service) where $\mathrm{X}$ can be Software, Platform or Infrastructure etc. Cloud users use these services provided by the Cloud service providers and build their application in the internet and thus deliver them to their end users. Thus cloud users need not to worry about the resources like hardware, software and maintenance of these infrastructures. They can also afford these cloud services as they are based on the pay-as-you-use. So the cloud users can reduce their expenditure and effort in the field of IT using cloud services instead of establishing IT infrastructure themselves.

Cloud is essentially provided by large distributed data centers. These data centers are often organized as grid and the cloud is built on top of the grid services. Cloud users are provided with virtual images of the physical machines in the data centers. This virtualization is one of the key concept of cloud computing as it essentially builds the abstraction over the physical system. Many cloud applications are gaining popularity day by day for their availability, reliability, scalability and utility model. These applications made distributed computing easy as the criticalaspects are handled by the cloud provider itself.

Cloud computing is growing now-a-days in the interest of technical and business organizations but this can also be beneficial for solving social issues. In the recent time EGovernance is being implemented in developing countries to improve efficiency and effectiveness of governance. This approach can be improved much by using cloud computing instead of traditional ICT. In India, economy is agriculture based and most of the citizens live in rural areas. The standard of living, agricultural productivity etc. can be enhanced by utilizing cloud computing in a proper way. Both of these applications of cloud computing have technological as well as social challenges to overcome.

Advantages of using Cloud - The advantages for using cloud services can be of technical, architectural, business etc. $[15,17]$.

i. Cloud Providers' point of view

(a) Most of the data centers today are underutilized. They are mostly $15 \%$ utilized. These data centers need spare capacity just to cope with the huge spikes that sometimes get in the server usage. Large companies having those data centers can easily rent those computing power to other organizations and get profit out of it and also make the resources needed for running data center (like power) utilized properly.

(b) Companies having large data centers have already deployed the resources and to provide cloud services they would need very little investment and the cost would be incremental.

ii. Cloud Users' point of view

(a) Cloud users need not to take care about the hardware and software they use and also they don't have to be worried about maintenance. The users are no longer tied to someone traditional system. (b) Virtualization technology gives the illusion to the users that they are having all the resources available.

(c) Cloud users can use the resources on demand basis and pay as much as they use. So the users can plan well for reducing their usage to minimize their expenditure.

(d) Scalability is one of the major advantages to cloud users. Scalability is provided dynamically to the users. Users get as much resources as they need. Thus this model perfectly fits in the management of rare spikes in the demand.

Motivation towards Cloud in Recent Time - Cloud computing is emerging technology, not a new idea but it is an evolution of an old paradigm of distributed computing. Recent technology trends and business models are few of the examples for enthusiasm about cloud computing in the corporate world [17] and scientific community across the world.

- High demand of interactive applications - Applications with real time response and with capability of providing information either by other users or by nonhuman sensors gaining more and more popularity today. These are generally attracted to cloud not only because of high availability but also because these services are generally data intensive and require analyzing data across different sources.

- Parallel batch processing - Cloud inherently supports batch-processing and analyzing tera-bytes of data very efficiently. Programming models like Google's mapreduce[18] and Yahoo!'s open source counterpart, Hadoop can be used to do these hiding operational complexity of parallel processing of hundreds of cloud computing servers.

- New trends in business world and scientific community In recent times the business enterprises are interested in discovering customer's needs, buying patterns, supply chains to take top management decisions. These require analysis of very large amount of online data. This can be done with the help of cloud very easily. Yahoo! Homepage is a very good example of such thing. In the homepage they show the latest news in the country. And according to the users' interest they change the ads and other sections in the page. Other than these many scientific experiments need very time consuming data processing jobs like LHC (Large Hadron Collider) which can be done by cloud.

- Extensive desktop application - Some desktop applications like Matlab, Mathematics, Cadenece etc. are becoming so compute intensive that a single desktop machine is no longer enough to run them. So they are developed to be capable of using cloud computing to perform extensive evaluations.

\section{Proposed Work}

Road traffic management involves directing vehicular and pedestrian traffic to ensure the safety of general public. The efforts by traffic managers and town planners to overcome traffic congestion are not achieving result as more and more vehicles are added to the road network day by day which exceeds the intended physical capacity of the road space. To 
tide over the traffic congestion and to ensure better traffic management, timely availability of dynamic traffic information with traffic managers are essential to regulate the traffic and divert the traffic to alternate routes.

There are several sources providing traffic information to commuters through FM stations, GPS assisted systems. However, these are not covering the entire segment of population as these systems have lot many disadvantages.

In this proposed work, we are presenting a new traffic management system infrastructure which will help in mitigating the traffic problems and ensure better traffic management, and efficient way to utilize time on the road. We are proposing a set of mobile applications i.e., an application for our clients, and another for the controller. The mobile application acts as an intermediate agent between the clients and the traffic management system. The aim is to provide a better solution for the commuter and the existing traffic management departments.

The figure 2 represents the proposed traffic management system. The system consists of client mobile application, controller mobile application, cloud network. The client mobile application is used by the registered clients who will access the traffic data from the cloud. The controller application is used by the set of controllers who will update the real time traffic data to the cloud. The cloud network consists of client database, controller database, and traffic database.

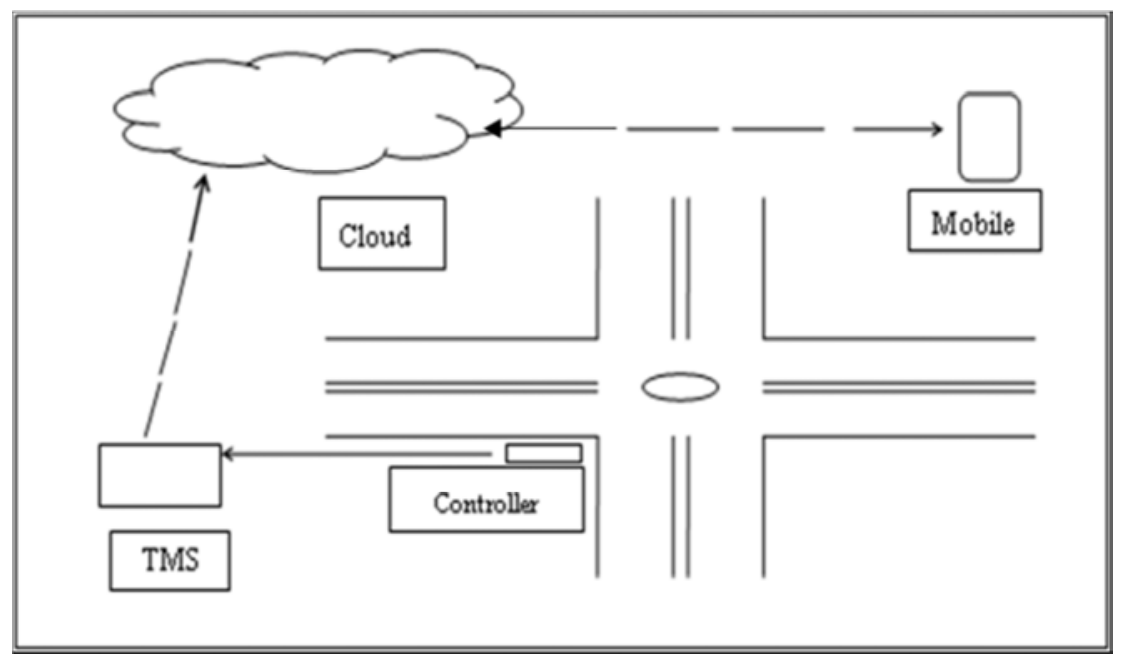

Figure 2. Proposed Model.

\subsection{Mobile Application}

Both the user mobile application and client mobile application are developed on the android operating system. The application is developed keeping the thoughts to make it easy to use and user friendly by simple user interface with minimal input. The mobile application has good security features and with the view of the launching of the application and taking this project to enterprise level we have included parts where user submits his personal data, which in turn helps us to expand the services. If it is required to be at premium level services that can be offered like, on demand parking slot in various parking spaces, on demand request of the quickest way to reach destination and so on. These premium services are provided on the extra payment and are not included in premium services.

\subsection{Client Application}

The main purpose of client application is to fetch the traffic data from the cloud. It consists of the signup page and login page.

A new user is required to enter the email, mobile number, desired password, and personal details to use the services. Once the user is successfully registered, the user is asked to login and then the user can access the data

\subsection{Controller Application}

The controller application is used by the set of registered users who act as controllers and who help in updating real time traffic data to the cloud networks.

A controller application has again signup page and the login page and then the easiest way to update the traffic by selecting the location id and stating the traffic status as listed.

\subsection{Cloud Network}

Cloud network plays a vital role in the application, as it stores various data regarding the clients, controllers and the traffic databases. In the cloud infrastructure we use WAMP Server consisting of Apache web server, OpenSSL for SSL support, MySQL database and PHP programming language.

\subsection{Client Database}

The information provided by the number of clients during the registration are stored using MySQL database. [android_api(database)-> users(tables)] Stores the information like Id, Unique_id, name, email, mobile number, location, date of birth, encrypted password, created_at(timestamp). And the same mobile number and the password is used for login purposes in the mere future. 


\subsection{Controller Database}

The information provided by the number of controller during the registration are stored using MySQL database. [android_api(database)->cont(tables)] stores the information like Id, Unique_id, name, email, mobile number, location, date of birth, encrypted password, created at(timestamp). And the same mobile number and the password is used for login purposes in the mere future.

\subsection{Traffic Database}

The predefined rows and columns in the traffic table defines the locations. These rows are accessed using the id's and are updated using update function. And hence for every update, an id with respective status column gets affected.

\subsection{Softwares Used}
a. Android Studio
b. Eclipse
c. WAMP Server

\subsubsection{Android Studio}

Android Studio is the official IDE for Android application development, based on IntelliJ IDEA. On top of IntelliJ's powerful code editor and developer tools, Android Studio offers even more features that enhance your productivity when building Android apps. [43]

\subsubsection{Eclipse}

Eclipse is an integrated development environment (IDE) used in computer programming. It contains base workspace and an extensible plug-in system for customising the environment. Eclipse is written mostly in java and its primary use is for developing java applications, but it may also be used to develop the applications in the other programming languages through the use of plug-ins, including: Ada, ABAP, C, C++, COBALT, Fortran, Haskell, JavaScript, Julia, Lasso, Lua, Perl, PHP, Python, Ruby etc. [44]

In our project we used Eclipse IDE for PHP programming.

\subsection{WAMP Server - Wamp Server}

Refers to a software stack for the Microsoft Windows operating system, created by Romain Bourdon and consisting of the Apache Web Server, Open SSL for SSL support, MySQL database and PHP programming language. [45]

\section{Results}

TRANSIRE is designed to be used when the user is mobile. A simple, intuitive user interface is developed. Moreover, the services and the features should be readily accessible and user should not need to spend time reading manuals to figure out how to use the basic functionality. This philosophy of simplicity, accessibility and user-friendliness is embodied in the design of the application.

The primary services provided by the TRANSIRE are accessible from the main page of the client's application which makes it quick and easy to navigate across particular traffic point in the city.

\section{Statistical Analysis}

The below graph in figure 3 describes the number of applications installed during the period of February 2016 to May 2016 and the number of active users during the same period too.

The below graph in figure 4 describes the traffic density at the Engineering College Circle. The data is updated by the controller using the controller application on 09/05/2016.

\section{Applications}

1. The application can be leased to Traffic police department or any other government

2. Services to monitor and scale the traffic on the roads. Thus being a part of digital India mission.

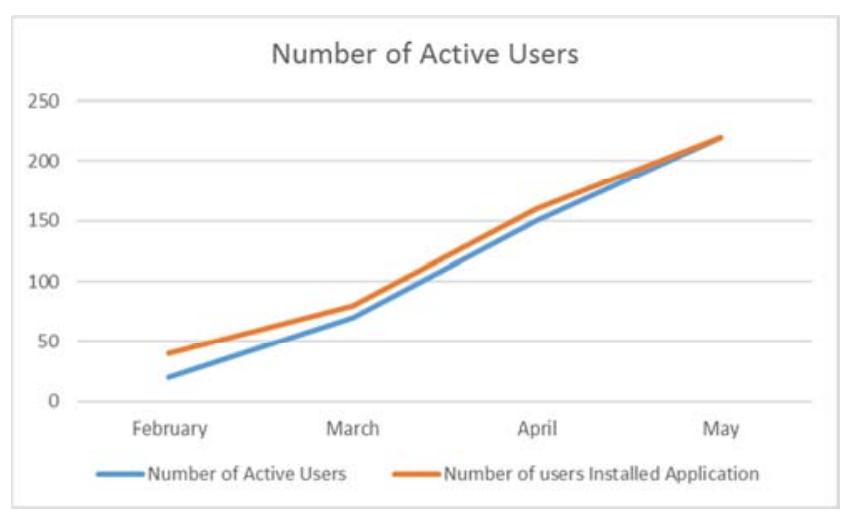

Figure 3. Number of applications installed versus number of users during a specific period.

3. The application can be leased to private logistic players to have a smooth transit services during their day to day operations.

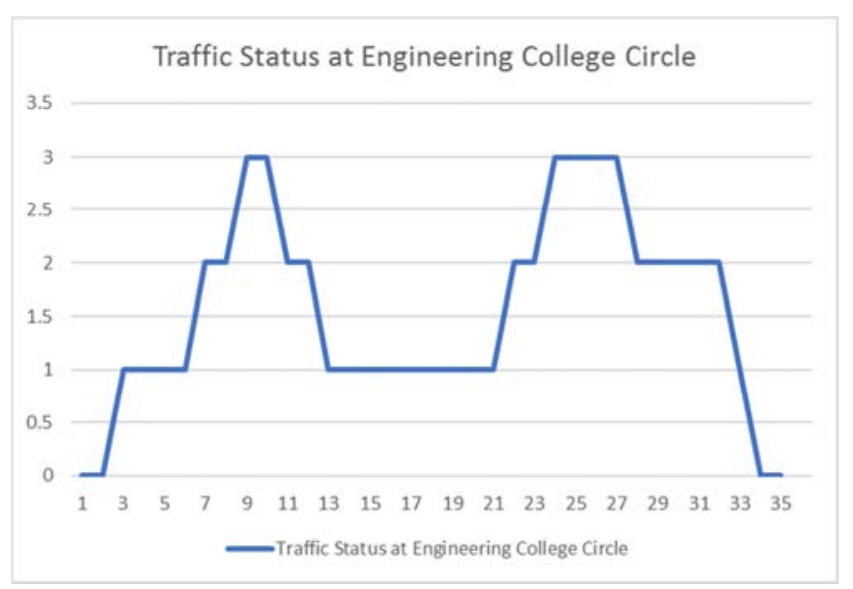

Figure 4. Traffic status at Engineering college.

4. A freemium application on the Google play store help in commuters to save their time during their travel.

5. Premium application helps in reducing time and fuel 
costs. There by indirectly helping a part of Green India mission

6. The same application can be used to monitor the traffic in the highways. Thus it can also be used by Highway patrol officers. Providing a smooth transit in national highways and expressways.

\section{Conclusion}

The project has aimed to solve the present and essential criteria of the people; travel has become unavoidable need of the people. To make the travel easy and fast we are come with a mobile application to find the traffic conditions of the critical points of a different city. Every application is different for different cities but they are clustered under a single head called Vehicular traffic management using cloud network. The people can download the application of their own cities and use for their comfort. By this the entrepreneurs, such as young engineers like us can earn money and give service. the expected results show the good performance of the project and some valid statistics.

\section{Future Work}

The project can be extended by using sensors and giving the automation to the application which needs manual services and software empowerment.

\section{References}

[1] Jan Holler et al., From Machine-to-Machine to the Internet of Things: Introduction to a New Age of Intelligence, 1st ed. London, United Kingdom: Academic Press Ltd, 10 Apr 2014.

[2] L. Atzori, A. Iera, G. Morabito, The Internet of Things: A Survey, Computer networks, 54(16), pp. 2787-2805, 2010.

[3] G. Tripathi, D. Singh, "EOI: Entity of Interest Based Network Fusion for Future Internet Services", ICHIT2011, September 23-25, 2011, Daejeon, Korea. (C) Springer-Verlag Berlin Heidelberg, CCIS, vol. 206, pp. 39-45, 2011.

[4] M. Zhang, T. Yu, G. F. Zhai, Smart Transport System Based on "The Internet of Things," Amm. 48-49 (2011) 1073-1076.

[5] M. Yun, B. Yuxin, Research on the architecture and key technology of Internet of Things (IoT) applied on smart grid, Advances in Energy Engineering (ICAEE). (2010) 69-72.

[6] H. Jun-Wei, Y. Shouyi, L. Leibo, Z. Zhen, W. Shaojun, A Crop Monitoring System Based on Wireless Sensor Network, Procedia Environmental Sciences. 11 (2011) 558-565.

[7] P. Kumar, S. Ranganath, W. Huang, K. Sengupta, Framework for real-time behavior interpretation from traffic video, IEE Transactions on Intelligent Transportation Systems. 6 (2005) 43-53.

[8] European Lighthouse Integrated Project - 7th Framework, Internet of Things - Architecture. http://www.iot-a.eu/ (2012).

[9] Kotis, K., and Katasonov, A. 2012. Semantic Interoperability on the Web of Things: The Smart Gateway Framework. In Proceedings of the Sixth International Conference on Complex, Intelligent, and Software Intensive Systems (CISIS2012), Palermo, 2012.

[10] Sheth, A., Henson, C., \& Sahoo, S. (2008), "Semantic Sensor Web," IEEE Internet Computing, July/August, p. 78-83.

[11] Jara, A. J., Varakliotis, S., Skarmeta, A. F., Kirstein, P. (2013), "Extending the Internet of Things to the Future Internet through IPv6 support". Mobile Information Systems, IOS Press.

[12] G. Gang, L. Zeyong, and J. Jun, "Internet of Things Security Analysis," in International Conference on Internet Technology and Applications (iTAP), August 2011.

[13] Z. Hu, "The research of several key question of Internet of Things," in International Conference on Intelligence Science and Information Engineering (ISIE), August 2011.

[14] Leonard Kleinrock. An internet vision: the invisible global infrastructure. Ad Hoc Networks, 1(1): 3-11, 2003.

[15] F. M. Aymerich, G. Fenu, and S. Surcis. An approach to a cloud computing network. Applications of Digital Information and Web Technologies, 2008. ICADIWT 2008., pages 113 118, August 2008.

[16] Bhaskar Prasad Rimal, Eunmi Choi, and Ian Lumb. A taxonomy and survey of cloud computing systems. Networked Computing and Advanced Information Management, International Conference on, 0: 44-51, 2009.

[17] Michael Armbrust, Armando Fox, Rean Griffith, Anthony D. Joseph, Randy H. Katz, Andrew Konwinski, Gunho Lee, David A. Patterson, Ariel Rabkin, Ion Stoica, and Matei Zaharia. Above the clouds: A berkeley view of cloud computing. Technical Report UCB/EECS-2009-28, EECS Department, University of California, Berkeley, Feb 2009.

[18] Ralf La"mmel. Google's mapreduce programming model revisited. Sci. Comput. Program., 70(1): 1-30, 2008.

[19] Samah Ahmed Zaki Hassan, "STAR: A Proposed Architecture for Cloud Computing Applications", International Conference on Cloud Computing, Technologies, Applications \& Management, pp-186-192, Dubai, 2012.

[20] Junjie Peng, Xuejun Zhang, Zhou Lei, Bofeng Zhang, Wu Zhang and Qing Li, "Comparison of several Cloud Computing Platforms", Second International Symposium on Information Science and Engineering, pp-23-26, 2009.

[21] Jiafu Wan, Di Li, Caifeng Zou and Keliang Zhou, "M2M Communications for Smart City: An Event-Based Architecture", IEEE 12th International Conference on Computer and Information Technology, pp-895-900, Guangzhou, 2012.

[22] Marta Santos, Ricardo Lopes Pereira and António Brandão Leal, "GBUS - Route Geo Tracer", IEEE $1^{\text {st }}$ International workshop on Vehicular Traffic Management for Smart cities, Dublin, 2012.

[23] Anutosh Maitra, Saurabh Bhadkaria, Chiranjeeb Ghosh, Sanjoy Paul, "An Integrated Transport Advisory System for Commuters, Operators and City Control Centres", IEEE $1^{\text {st }}$ International workshop on Vehicular Traffic Management for Smart cities, Dublin, 2012. 
[24] Claudia Campolo, Antonio Iera, Antonella Molinaro, Stefano Yuri Paratore, Giuseppe Ruggeri, "SMaRTCaR: An Integrated Smartphone-based Platform to Support Traffic Management Applications", IEEE $1^{\text {st }}$ International workshop on Vehicular Traffic Management for Smart cities, Dublin, 2012.

[25] Vi Tran Ngoc Nha, Soufiene Djahel and John Murphy Lero, "A Comparative Study of Vehicles' Routing Algorithms for Route Planning in Smart Cities", IEEE $1^{\text {st }}$ International workshop on Vehicular Traffic Management for Smart cities, Dublin, 2012.

[26] Longzhao Zhong, Beizhan Wang and Haifang Wei, “Cloud Computing Applied in the Mobile Internet", IEEE 7th International Conference on Computer Science and Education, pp-218-221, Melbourne, Australia, 2012.

[27] Tareq Binjammaz, Ali Al-Bayatti, Ashwaq Al-Hargan, "GPS Integrity Monitoring for an Intelligent Transport System", IEEE $10^{\text {th }}$ workshop on Positioning Navigation and Communication, Dresden, 2013.

[28] Joydeep Paul, Baljeet Malhotra, Simon Dale, Meng Qiang, "RFID Based Vehicular Networks for Smart Cities", 29 International Conference on Data Engineering Workshops, pp120-127, Brisbane, QLD, 2013.

[29] Mehdi Bahrami, "Cloud Computing for Emerging Mobile Cloud Apps", IEEE $3^{\text {rd }}$ International Conference on Mobile Cloud Computing, Services, and Engineering, pp-4-5, San Francisco, 2015.

[30] Deepak Puthal, B. P. S. Sahoo, Sambit Mishra, and Satyabrata Swain, "Cloud Computing Features, Issues and Challenges: A Big Picture", IEEE International Conference on Computational Intelligence \& Networks, pp-116-123, Bhubaneshwar, IN, 2015.

[31] Andrea Zanella, Nicola Bui, Angelo Castellani, Lorenzo Vangelista, "Internet of Things for Smart Cities", IEEE INTERNET OF THINGS JOURNAL, VOL. 1, NO. 1, pp-22$32,2014$.

[32] Carolina Tripp Barba, Miguel A' ngel Mateos, Pablo Regan as Soto, Ahmad Mohamad Mezher and M'onica Aguilar Igartua, "Smart city for VANET's using warning messages, traffic statistics and intelligent traffic lights", Intelligent Vehicles Symposium Alcalá de Henares, Spain, pp-902-907, 2012.

[33] Mr. V. Shashikiran, T. T Sampath Kumar, N. Sathish Kumar, V. Venkateshwaram, S Balaji "Dynamic Road Traffic Management based on Kruskal's Algorithm", International Conference on recent trends in Information techonology pp. 200 - 204, Chennai, 2011.
[34] Li Yuewen and Wu Zhong, "Research of Intelligent Model of Optimal Route for the Urban Public Transport", IEEE $2^{\text {nd }}$ International Conference on Business Computing and Global Informatization, pp-695-698, Shanghai, 2012.

[35] Pinku Hazarika, Vinod Baliga, Seshubabu Tolety, "The Mobile-Cloud Computing (MCC) Roadblocks", IEEE $11^{\text {th }}$ International Conference on Wireless and Optical Communications Networks, Vijayawada, AP, IN, 2014.

[36] Nicholas Loulloudes, George Pallis, Marios D. Dikaiakos, " $V$ Radar: A Vehicular Traffic Query Protocol for Urban Environments", IEEE $1^{\text {st }}$ International workshop on Vehicular Traffic Management for Smart cities, Dublin, 2012.

[37] Soufiene Djahel, Ronan Doolan, Gabriel-Miro Muntean, and John Murphy, "A Communications-Oriented Perspective on Traffic Management Systems for Smart Cities: Challenges and Innovative Approaches", IEEE COMMUNICATION SURVEYS \& TUTORIALS, VOL. 17, NO. 1, pp-125-151, 2015.

[38] Dhananjay Singh, Gaurav Tripathi, Antonio J. Jara, “A survey of Internet-of-Things: Future Vision, Architecture, Challenges and Services", IEEE World Forum on Internet of Things (WFIoT), pp. 287-292, 2014.

[39] Teemu Leppanen, Arto Heikkinen, Antti Karhu, Erkki Harjula, Jukka Riekkiand Timo Koskela, "Augmented Reality Web Applications with Mobile Agents in the Internet of Things", Eigth International Conference on Next Generation Mobile Applications, Services and Technologies, pp. 54-59, 2014.

[40] Rafiullah Khan, Sarmad Ullah Khan, Rifaqat Zaheerand Shahid Khan, "Future Internet: The Internet of Things Architecture, Possible Applications and Key Challenges", 10th International Conference on Frontiers of Information Technology, pp. 257-260, 2012.

[41] Mohsen Hallaj Asghar, Nasibeh Mohammadzadeh, Atul Negi, "Principle Application and Vision in Internet of Things (IoT)", International Conference on Computing, Communication and Automation, pp. 427-431, 2015.

[42] Louis COETZEE, Johan EKSTEEN, "The Internet of ThingPromise for the Future? An Introduction", IIMC International Information Management Corporation, pp. 1-9, Africa, 2011.

[43] http://developer.android.com/

[44] https://en.wikipedia.org/wiki/Eclipse_(software)

[45] https://en.wikipedia.org/wiki/WampServer 